\title{
How to Improve the Reliability of Electronic Components
}

Liu Miao Wu Wei Wei Menglin Chen Zhimin

Institute of Metrology and Measurement Technology, Hubei Aerospace Science and Technology Institute, Xiaogan 432000

Abstract: People's daily life are inseparable from high-tech electronic products. Many high-tech manufacturing industries face a huge number of production orders every day, in order to meet the huge demand many manufacturers in the production process ignored the quality of electronic components. There have been many high-tech products using low-quality electronic components, resulting in a series of quality problems. So the application of electronic components to enhance the quality of this task is imminent. China's related enterprises and staff are also constantly improve the quality of electronic components to reduce the emergence of system equipment failure problems. The following article on the promotion of its reliability of the relevant content were described for reference.

Key words: electronic components; reliability; strategy

\section{Introduction}

With the rapid development of social modernization technology, high-tech products are constantly innovation. One of the electronic components is the production of these high-tech products, the main material, it is in the development of modern technology cannot shake the important position. For the quality of electronic components to control the work cannot be wrong. If you cannot guarantee that electronic components have a good quality assurance, it will be on the high-tech products manufacturing industry has brought immeasurable serious consequences.

\section{The importance of electronic components}

Strengthen the quality of electronic components analysis and control can improve the quality of electronic components to prevent some of the poor quality of electronic components into the production line. So that China's high-tech industry can create a better production environment. And the quality analysis and control of electronic components can help enterprises with less financial expenses. To avoid the production of product quality, but related to the issue of compensation. On the surface in the detection equipment to increase the capital investment, but in fact the production of electronic components of excellent quality, low return rate for enterprises to bring greater profits. The inherent quality of the components is mainly determined by the production unit of the components in the selection of raw materials, components design, process, manufacturing and other quality control process; components of the use of quality is mainly by the choice of components, procurement, Screening, testing and use of the user in the process of quality control under the decision ${ }^{1}$.

\section{Key factors affecting the quality of electronic components used}

\subsection{Design factors}

There are many reasons for the failure of electronic components, through the specific analysis of these reasons, you can find the impact of the use of electronic components of the most important factor is the design factor. According to the survey, the electronic components of the design unit can be learned, the design unit of the design of electronic components in the process, the design staff of the lack of practical understanding of electronic equipment, ignoring the test of electronic components, but also the lack of science system settings ${ }^{2}$. In addition, the design unit and the use of units without timely 
communication, which makes the design unit cannot quickly receive feedback on the use of information on the electronic components of the problems cannot be amended in a timely manner, resulting in long-term design problems.

\subsection{Management factors}

First of all, many enterprises producing electronic components did not develop product quality standards, resulting in the production process for product quality without standard evaluation requirements. Resulting in a lot of great differences in the quality of electronic components. Secondly, many companies produce electronic components of the technical means of less than the national standard. This poses a great deal of difficulty in the analysis and control of electronic components. The last point is that in the production of electronic components in the process of many staff cannot correct their own work attitude, negative attitude, loose. Which also led to the production of electronic components there are serious quality problems ${ }^{3}$.

3. To improve the reliability of the use of electronic components measures

\subsection{Combined with the practical application of electronic components}

At present, China's production of electronic components and no unified management, so the market of electronic components of the specifications, logo, etc. are very different. So the use of units on the production of manufacturers do not have much understanding, so the parameters of components such as no good understanding, thus affecting the actual application effect. So manufacturers in the purchase of components in the process, to the different logo of the plant have a certain understanding of the components of the real information can be determined, the manufacturers of products to compare, and thus buy the best quality components in the purchase process, companies can follow two principles: 1. Buy national certification and complete the certificate components; 2 . With the credibility of the technology mature manufacturers.

\subsection{To enhance the overall quality of components management staff}

A variety of electronic components, the key performance parameters of each product. This requires managers to understand the technical level of components and trends, foreign parts manufacturers and their products, but also familiar with the domestic scientific research units and product status parts; basic conditions, technical features not only familiar with and require the type of components, but also understand the relevant performance indicators of various components, product quality level, packaging, size and supply support capabilities. Component management must have a strong sense of responsibility, must have a wealth of expertise, organizational coordination and high-quality comprehensive talent.

\subsection{Good components of the screening work}

Reduce the use of electronic components in the process of the phenomenon of the phenomenon, the relevant enterprises should strengthen the screening of electronic components, so that electronic components in the equipment and system applications to meet the standard requirements, the enterprise can be selected from the following aspects: 1 . Detection work, testing and circuit testing are complementary, usually the use of the judge circuit parameters are qualified to determine the use of electronic components. According to this test can reflect the performance of electronic components, reflecting the situation and a series of problems, which can effectively prevent the hidden dangers of electronic components; 2. Strict screening process: In the process of its inspection, timely processing the problem of electronic components, replacement does not meet the requirements, to ensure the quality of electronic equipment applications ${ }^{4}$.

\subsection{To strengthen the inspection standards for electronic components}

In the electronic components after the end of the production process, but also to strengthen the test for the production of products. To go through multiple steps. First, the production of electronic components manufacturers to test. In the production of electronic components to ensure the quality of qualified after the second batch of inspection. In order to meet the electronic components can be put into use, will be the second batch of quality inspection, the quality of electronic components to reduce the risk of quality problems. In the double test under the standard according to the special needs of the product more advanced screening and testing to meet the enterprise for some special requirements of electronic components 5. After experiencing a number of stringent inspection standards can make the quality of electronic components to be well protected. So as to meet the production needs of enterprises. 
In short, it can be seen that electronic components in electronic equipment is an important component, but the practice has proved that there are many problems in the application process, so the relevant enterprises should strengthen their supervision, so that the use of the problems get timely solution, to avoid affecting the normal operation of electronic equipment. Production businessmen should also fundamentally innovative design, and constantly improve the function of electronic components to enhance its reliability.

\section{References}

[1] Wang Zili. Analysis of Electronic Components Quality and Reliability Management [J]. Communication world, 2016, 21: 216-217.
[2] Wu Dongfeng. Key Technology and System for Quality Control of Electronic Components [D]. Beijing University of Science and Technology, 2015.

[3] Zhu Dongsheng, Lei Junxi, Wang Changhong, Hu Hanying. Research Progress of Thermoelectric Cooling Technology for Electronic Components [J]. Microelectronics, 2009, 01: 94-100.

[4] Xue Haili, Guo Tao, Wang Hui. Selection and Use of Military Electronic Components [J]. Torpedo Technology, 2005, 01: 33-36.

[5] Huang Suping. Electronic Components Reliability and Detection Screening [J]. China's new technology and new products, 2010, 04: 143. 